\title{
Adaptive Parameter Estimation of IIR System-Based WSN Using Multihop Diffusion in Distributed Approach
}

\author{
Meera Dash, ITER, Siksha 'O’ Anusandhan (Deemed), Bhubaneswar, India \\ Trilochan Panigrahi, National Institute of Technology Goa, India \\ Renu Sharma, ITER, Siksha 'O' Anusandhan (Deemed), Bhubaneswar, India \\ Mihir Narayan Mohanty, ITER, Siksha 'O’ Anusandhan (Deemed), Bhubaneswar, India
}

\begin{abstract}
Distributed estimation of parameters in wireless sensor networks is taken into consideration to reduce the communication overhead of the network which makes the sensor system energy efficient. Most of the distributed approaches in literature, the sensor system is modeled with finite impulse response as it is inherently stable. Whereas in real time applications of WSN like target tracking, fast rerouting requires, infinite impulse response system (IIR) is used to model and that has been chosen in this work. It is assumed that every sensor node is equipped with IIR adaptive system. The diffusion least mean square (DLMS) algorithm is used to estimate the parameters of the IIR system where each node in the network cooperates themselves. In a sparse WSN, the performance of a DLMS algorithm reduces as the degree of the node decreases. In order to increase the estimation accuracy with a smaller number of iterations, the sensor node needs to share their information with more neighbors. This is feasible by communicating each node with multi-hop nodes instead of one-hop only. Therefore the parameters of an IIR system is estimated in distributed sparse sensor network using multihop diffusion LMS algorithm. The simulation results exhibit superior performance of the multihop diffusion LMS over non-cooperative and conventional diffusion algorithms.
\end{abstract}

\section{KEYWORDS}

Diffusion LMS, Distributed Estimation, IIR Systems, LMS, Multihop Diffusion, Parameter Estimation, Sparse Network, Wireless Sensor Network

\section{INTRODUCTION}

Wireless sensor networks (WSNs) is the most widely adaptive network comprising of a number of sensor nodes scattered in a topology for specific sensing implementations (Li \& Thai, 2008; Panda et. al., 2020). In the network each node tries to improve the estimates of the desired parameters taking into account its own and shared information from each and every sensor node in the network. The distributed estimation can be described mathematically as the optimization of a cost function which depends on all the information in a network (Panigrahi et al., 2016). For this, each sensor node after sensing the environment, processes the data and then sends it to a subset of the neighboring nodes. Therefore, great effort is required to formulate distributed estimation algorithms. The algorithm using the distributed approach improves the estimate of the parameters in each sensor node and minimizing the communication overhead thus providing energy efficiency to the system(Sayed, 2014b). 
In case of sparse wireless sensor network or less connected networks the nodes are scattered farapart in a geographic area. The number of neighbors participating in data aggregation and sharing for each node is less and even less for the nodes that lie at the edge of the network. These nodes face the difficulty in accessing the information from the network required for estimating the parameters(Kong, Lee, Kim, Shin, \& Song, 2017; Nayak, Panigrahi, \& Sharma, 2015). In this scenario one of the solutions to improve the performance may be the centralized approach. But the major drawback of this approach is that it requires more number of communications between the nodes and the central processor. Thus it is not energy efficient as more energy is consumed in communication in comparison to processing(Bazzi, Rastegarnia, \&Khalili, 2014). In order to overcome these problems and to make the sensor syste m energy efficient, distributed parameter estimation approaches are proposed by authors in literature. There are two ways node can cooperate: incremental and diffusion.

Incremental cooperative approach was proposed for estimation of parameter with sharing information (Majhi\& Panda, 2013) using particle swarm optimization algorithm. This mode of operation requires a cyclic path among the sensor nodes which is not feasible in large and sparse WSNs (Khalili, Rastegarnia, Bazzi, \&Rahmati, 2017). The convergence speed is very slow and the algorithm is not adaptive in real time(Sayed, 2014b). Whereas in the diffusion cooperative strategy, every node updates the estimate and shares it with its one-hop immediate neighbors (Sayed, 2014a). This method in comparison to the incremental approach is adaptive to real time changes in the environment. In most of the literature, the distributed least mean square algorithm or its variants are used to estimate the parameters of the FIR system as it is inherently stable. But FIR systems need a greater number of parameters to have equivalent model of a real system. In fact, a feedback system may not be modeled exactly by using FIR even though with very high order. But the same feedback system can be modeled exactly by using IIR system. In WSNs, there are certain applications where the sensor nodes are modeled as IIR system. In this paper, we have considered an adaptive IIR system at each node. The Diffusion LMS algorithm is used to estimate the parameters of the adaptive IIR system with cooperation.

Further, the diffusion LMS algorithm for IIR system is extended to estimate the parameters in sparse sensor network. In sparse network where the nodes are connected to a smaller number of neighbors, the estimation performance degrades and need a greater number of iterations to achieve the steady state value which leads to the algorithm energy inefficient. Therefore, multi-hop diffusion LMS is proposed here where a sensor node communicate with more than one hop sensor nodes unlikely only to one hop in diffusion LMS algorithm (Hu \&Tay, 2015; Kong et al., 2017).

Multi-hop diffusion LMS is proposed here for a sparse WSN to estimate IIR system parameters and the estimates are compared with the performances of incremental LMS and non-cooperative LMS algorithm. The efficient data aggregation protocol in WSNs is used for multi-hop (Mohapatra \&Majhi, 2017; Xu et al., 2009). The simulation results show that the proposed multi-hop diffusion strategy outperforms the conventional non-cooperative LMS and incremental LMS algorithms strategies. In fact, it may be thought that in multi-hop diffusion the communication overheads increase by $h$ times. But in comparison to conventional diffusion strategy the proposed multi-hop diffusion strategy requires lesser iterations to achieve the steady state. This is because each sensor gets more information to update its own estimates. The simulation results of different algorithms are compared with respect to mean square error and mean square deviation taking into consideration a number of standard benchmarks IIR systems.

The paper has been organized into 5 Sections. The $2^{\text {nd }}$ Section is about the parameter estimation by distributed diffusion mode of cooperation, Section 3 describes the distributed estimation using multi-hop diffusion strategy, $4^{\text {th }}$ Section is results and discussion. Section 5 is the conclusion of the work with future work. 


\section{DISTRIBUTEDPARAMETER ESTIMATION BY DIFFUSION MODE OF COOPERATION}

In this section we have described the estimation of IIR systems parameter using diffusion cooperation in sensor network. Let us consider a sensor network with $N$ number of sensor nodes distributed in a geographical area. The sensor nodes are interconnected and have its topology. The nodes are assumed to be communicating to the neighboring nodes with standard communication protocol (Panigrahi et al., 2016).

Each node in the sensor network is now modeled as IIR systems (varying in time) with $P$ and $Q$ number of poles and zeros respectively. The input-output of the system is modeled as in (Agrawal, Kumar, Bajaj, \& Singh, 2017).In the sensor network, each node $k$ has noisy output $d_{k}(i)$ to the input data vector $x_{k}(i)$ at each time instant $i$. The noise is assumed to be independent of both the input and output of the IIR system. Hence the observations obtained at each node are both independent spatially and temporally. Here $v_{k}(i)$ refers to the noise measured at the $i^{\text {th }}$ time instant of the $k^{\text {th }}$ sensor node with zero mean and variance $\sigma_{v, k}^{2}$. The noise here is taken to be normalized white Gaussian which is uncorrelated with the input data. In this case each node is considered to be IIR systems with same number of poles and zeros. At each node the estimated output can be written as a recursive equation as given in (1) (Dash et al. 2019),

$$
d_{k}(i)=\sum_{p=1}^{P} a_{p} d_{k}(i-p)+\sum_{q=0}^{Q} b_{q} x_{k}(i-q)+v_{k}(i)
$$

Where $\left\{a_{p}\right\}_{p=1}^{P}$ and $\left\{b_{q}\right\}_{q=0}^{Q}$ are the pole and zero parameter of the IIR filter and $v_{k}(i)$ is the noise measured at the $i$ th time instant of the $k$ th sensor node with zero mean and variance $\sigma_{v, k}^{2}$. To attain the desired steady state the estimated coefficients at each sensor node is adjusted during training. To estimate the global parameter vector $w$ using the information $\left\{d_{k}(i), x_{k}^{i}\right\}$, available at each sensor node by following the adaptive distributed optimization (Nedic \& Ozdaglar, 2009) is the main objective of this paper. Optimizing the given cost function to estimate $w$ (the optimum least square estimation vector).

$$
\begin{aligned}
& C(w)=\sum_{k=1}^{K} E\left\|d_{k}(i)-u_{k}^{i} w\right\|^{2} \\
& C(w)=\sum_{k=1}^{K} C_{k}(w)
\end{aligned}
$$

Where $C_{k}(w)=E\left\|d_{k}(i)-u_{k}^{i} w\right\|^{2}$ is the local cost function. The optimal least square solution is obtained by solving Equation (3).

$$
\hat{w}=\arg \min \sum_{k=1}^{K} E\left\|d_{k}(i)-u_{k}^{i} w\right\|^{2}
$$


The approximation of the mean squared error (MSE) leads to the popular LMS algorithm for FIR filter which can be further applied for IIR filter to estimate the desired response $d_{k}(i)$ (Dash et al., 2017). Here the diffusion LMS algorithm using the adapt-then-combine adaptive strategy is used to estimate the parameters of the IIR filter (Cattivelli \& Sayed, 2009; Das et al., 2017).

$$
\begin{aligned}
& e_{k}(i)=d_{k}(i)-u_{k}^{i} w_{k}^{i-1} \\
& \hat{w}_{k}^{i}=\hat{w}_{k}^{i-1}+\mu_{k} e_{k}(i) u_{k}^{i^{T}} \\
& \hat{w}_{k}^{i}=\sum_{l \in N_{k}} c_{l k} \hat{w}_{l}^{i}
\end{aligned}
$$

Where $\hat{w}_{k}^{i}$ is the estimate of the optimal parameter at sensor node $k$. It also has access to its neighbors estimates. The scalars $\left\{c_{l k}\right\}$ are positive weights which the sensor node $k$ assigns to the estimates of its neighbor nodes $N_{k}$. Weights satisfying,

$$
c_{l k} \geq 0, \sum_{l \in N_{k}} c_{l k}=1, \text { and } c_{l k}=0 \text { for } l \notin N_{k}
$$

Thus, each node in the sensor network cooperates among them by incorporating the above diffusion algorithm to estimate the IIR system's parameters.

\section{DISTRIBUTED ESTIMATION USING MULTIHOP DIFFUSION STRATEGY}

In literature, many ways have been proposed to improve the performance of distributed diffusion strategy in varied environments. Almost all of the authors in the literature have tried to reduce the communication overhead in the network because more energy is required for communication than computation (Arablouei, Werner, Huang, \& Doğançay, 2013; Gharehshiran, Krishnamurthy, \& Yin, 2013; Panigrahi, Pradhan, Panda, \& Mulgrew, 2012). But these methods failed to provide desired performance in a sparse (less connected) network. It is seen that the number of communications drastically reduces by using partial diffusion method (Arablouei et al., 2013). Energy aware distributed diffusion is discussed in (Gharehshiran et al., 2013). These methods too, are not suitable for IIR system parameter estimation problems. It is because the poles play an important role in IIR system and slight deviation in the value during the learning process may cause instability.

Recently, energy constrained multihop diffusion LMS is proposed in (Hu \& Tay, 2015; Nayak et al., 2015). But the physical 1-hop neighbor and the multihop or h-hop neighbors are defined as per the energy of each sensor node. The challenge is in aggregation of data from all the nodes. This multihop diffusion process is time consuming as for every iteration, the sensor nodes have to find the energy information of their neighbors.

Here, we have proposed a multihop diffusion adapt-then-combine (ATC) strategy without constraints. In comparison to the combine-then-adapt strategy (CTA) the ATC is more adaptive. Each sensor node in the network has the information from h-hop neighbor nodes in the network. The h-hop neighbors of the $k$ th node is defined as the set of sensor nodes present at a distance h-hop from itself. The topology for 1-hop and 2-hop neighbors is described in (Nayak et al., 2015).

$$
i=1,2, \ldots, K ; \hat{w}_{k}^{0}=w_{\text {init }}
$$




$$
\begin{aligned}
& \hat{w}_{k}^{i}=\hat{w}_{k}^{i-1}+\mu_{k} e_{k}(i) u_{k}^{i T} \\
& \hat{w}_{k}^{i}=\sum_{l \in N_{k}^{h}} c_{l k} \hat{w}_{l}^{i}
\end{aligned}
$$

Where $\mu \mathrm{k}$ is the step-size parameter of node $k$, and $\left\{c_{l k}\right\}$ are combination weights satisfying

$$
c_{l k} \geq 0, \sum_{l \in N_{k}^{h}} c_{l k}=1 \text {, and } c_{l k}=0 \quad \text { if } l \notin N_{k}^{h}
$$

The only difference between conventional ATC (5) and hATC (7) is in the combination step. In hATC each sensor node k aggregate information from h-hop neighbors $N_{h}{ }^{k}$ where the immediate neighbor $N^{k}$ is a subset. Whereas, in ATC, the sensor node $k$ receives information only from immediate neighbor. The combiner coefficients are same for both the strategies.

But, in distributed signal processing in sensor network, each sensor is assumed to be communicated only with 1-hop neighbors only. So, it is difficult to combine the estimates from multihop neighbors in (8). This can be calculated by repeating 1-hop combination $h$ times in $h$-hop diffusion strategy. The multihop algorithm can be implemented with the same communication protocol used in conventional diffusion LMS. The algorithm also does not require additional storage capacity. But the algorithm needs $(|\mathrm{Nk}|-1) h$ communication cost in each iteration. This is $h$ times more than the communication cost for DLMS ( $|\mathrm{Nk}|-1)$.

\section{RESULTS AND DISCUSSIONS BASED ON SIMULATION}

In this section, the simulation results of diffusion LMS algorithms are compared with conventional centralized algorithms and sequential cooperation among the nodes known as incremental LMS. In sparse sensor network, multihop diffusion LMS (hDLMS-IIR) is used. For simulation purposes, standard IIR systems are considered. The transfer function of $2^{\text {nd }}, 3^{\text {rd }}$ and $4^{\text {th }}$ order IIR system is given as (Dash et al., 2017). We have used $2^{\text {nd }}, 3^{\text {rd }}$ and $4^{\text {th }}$ order adaptive filter at each sensor node to model the $2^{\text {nd }}, 3^{\text {rd }}$ and $4^{\text {th }}$ order IIR system respectively. The average over 500 independent experiments are plotted.

\subsection{Comparison of Mean Squared Error and Mean Squared Deviation Performances}

The mean square error (MSE) and mean square deviation (MSD) performances for $2^{\text {nd }}, 3^{\text {rd }}$ and $4^{\text {th }}$ order systems using DLMS-IIR, ILMS-IIR and non-cooperative LMS-IIR algorithms are plotted in Figures 1-3. Different step sizes $\mu$ were chosen for different algorithm to keep the same convergence speed. The step sizes $0.055,0.015$ and 0.075 are chosen for non-cooperative LMS-IIR, ILMS-IIR and DLMS-IIR algorithms respectively. MSD at the $k^{\text {th }}$ sensor node in $i^{\text {th }}$ time instant is calculated as $\xi_{k}(i)=\left\|w^{o}-\hat{w}_{k}(i)\right\|^{2}$, where $w^{o}$ is the actual parameters and $\hat{w}_{k}(i)$ is the estimated parameter at the $k$ th sensor in the $i$ th time instant. MSD gives how closed the estimated parameter with optimum value during the learning time.

From Figures 1-3, it is clear that the diffusion LMS algorithm exhibits superior performance by providing less steady state MSE and MSD with same convergence speed compared to ILMS-IIR and non-cooperative LMS-IIR algorithms. The LMS-IIR provides poor performance because each sensor node tries to estimate the systems parameters by its own data. Whereas by doing cooperation in ILMS and DLMS algorithms the sensor nodes get more information in the network which leads 
Figure 1. Average MSE(a) and MSD(b) performances of $2^{\text {nd }}$ order IIR system by DLMS-IIR, ILMS-IIR and non-cooperative LMS-IIR algorithms

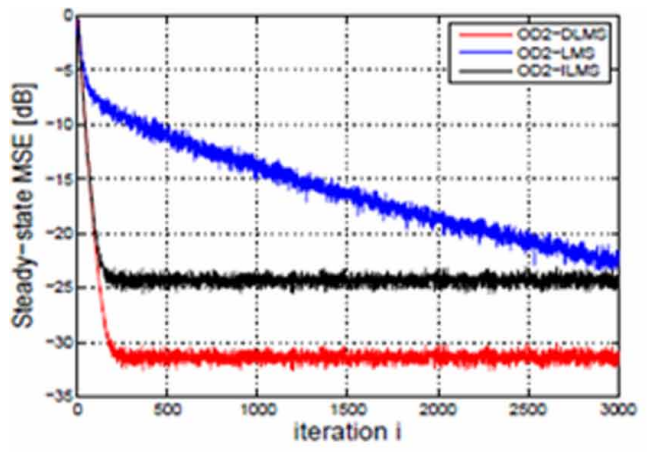

a

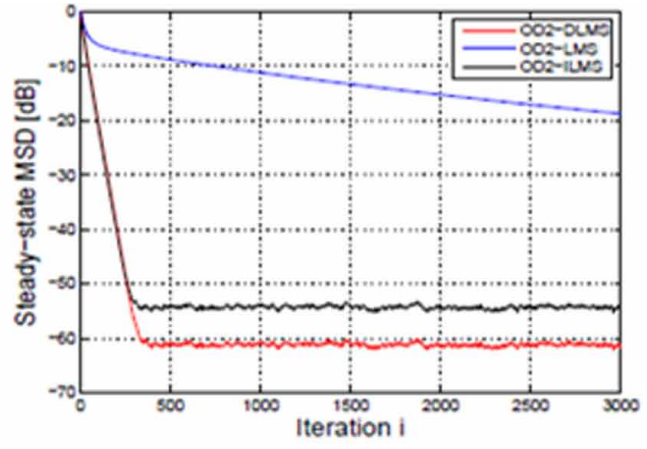

b

Figure 2. Average MSE (a) and MSD(b) performances by DLMS-IIR, ILMS-IIR and non-cooperative LMS-IIR algorithms for 3rd order IIR system.

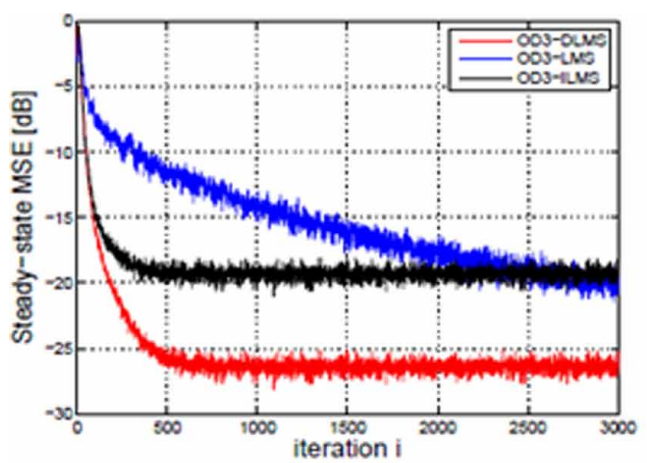

a

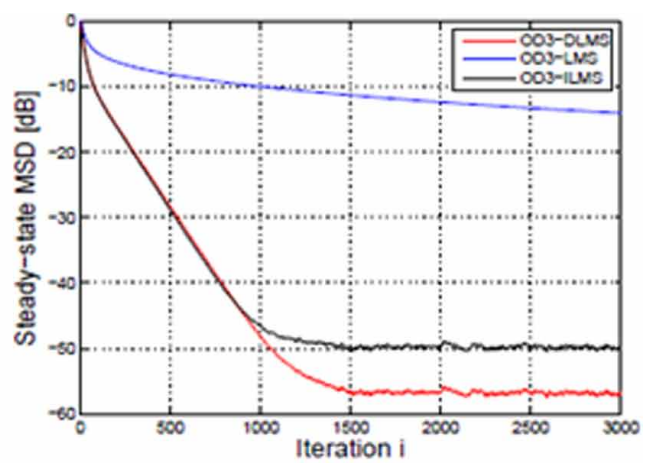

b

Figure 3. Average MSE (a) and MSD (b) performances of $4^{\text {th }}$ order IIR system by DLMS-IIR, ILMS-IIR and non-cooperative IIR algorithms.

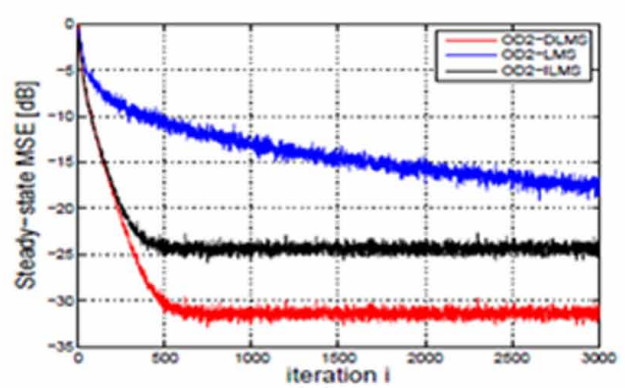

a

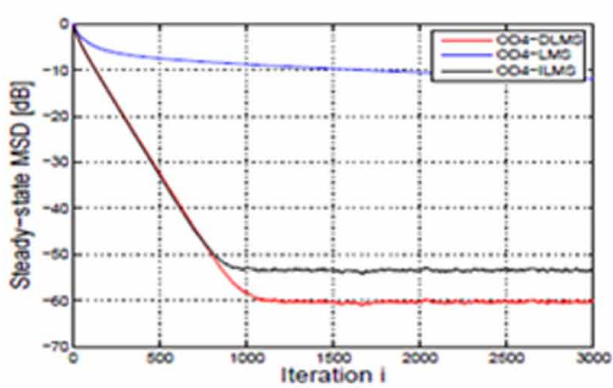

b 
to better estimation accuracy. At each iteration, each sensor nodes received updated information from the neighbor which is to be updated using local observation. The DLMS-IIR provides better MSD and MSE performance in comparison to the ILMS-IIR. It is because in the diffusion mode of cooperation, each node gets the information from all one-hop immediate neighbors. Then the sensor node combines the h-hop information and the local data to update the estimate. Whereas, in incremental mode, every sensor node gets information from one of its 1-hop immediate neighbor only. This information along with the local observed data is used for updating the estimate. Thus, the amount of data communication involved in DLMS is more than that of ILMS. But DLMS is adaptive to real time change in the network in comparison to ILMS and to define a cyclic path in the network is very difficult as is a requirement in ILMS.

Table 1. Estimated Parameters by DLMS-IIR, ILMS-IIR and non-cooperative LMS-IIR algorithms

\begin{tabular}{|c|c|c|c|c|}
\hline \multirow[t]{2}{*}{ System Order } & \multirow[t]{2}{*}{ Actual Parameter } & \multicolumn{3}{|c|}{ Estimated Parameters } \\
\hline & & LMS-IIR & ILMS-IIR & DLMS-IIR \\
\hline \multirow[t]{4}{*}{ 2nd } & 1.25 & 1.1851 & 1.2499 & 1.25 \\
\hline & -0.25 & -0.2116 & -0.2503 & -0.25 \\
\hline & 0.3 & 0.2954 & 0.3 & 0.3 \\
\hline & -0.4 & -0.3902 & -0.3999 & -0.3999 \\
\hline \multirow[t]{5}{*}{$3 \mathrm{rd}$} & 0.6 & 0.557 & 0.6001 & 0.6 \\
\hline & -0.4 & -0.3686 & -0.3988 & -0.4 \\
\hline & -0.2 & -0.2246 & -0.1954 & -0.2 \\
\hline & 0.5 & 0.4465 & 0.5 & 0.5 \\
\hline & -0.1 & -0.1231 & -0.1 & -0.1001 \\
\hline \multirow[t]{8}{*}{ 4th } & 1 & 0.8846 & 1 & 1 \\
\hline & -0.9 & -0.7529 & -0.9 & -0.9 \\
\hline & 0.81 & 0.7058 & 0.81 & 0.81 \\
\hline & -0.729 & -0.6857 & -0.7289 & -0.729 \\
\hline & -0.04 & -0.1409 & -0.401 & -0.0401 \\
\hline & -0.2775 & -0.2348 & -0.2275 & -0.2775 \\
\hline & 0.2101 & 0.2107 & 0.21 & 0.2101 \\
\hline & -0.14 & -0.14 & -0.1401 & -0.1401 \\
\hline
\end{tabular}

Comparing the performances of MSE and MSD of different algorithms taken into consideration, it is found that the values by using DLMS-IIR is less in comparison to other algorithms. Thus comparison of actual and estimated parameters have been illustrated in Table 1, which shows that parameters estimated by DLMS-IIR algorithm is nearly same to that of the actual parameters in comparison to that by ILMS-IIR and LMS-IIR algorithms. This shows that MSE and MSD performances by these algorithms. Thus, it can be said that DLMS-IIR algorithm is a good candidate for distributed IIR system parameter estimation. The APD and SE performances are measured at each node by using all the algorithms which is discussed in the next section. 


\subsection{Average Percentage of Deviation and Sum of Error Square Performance}

To evaluate the closeness of the estimated values with the actual parameters, the sum of error squares (SE) and average percentage of deviation (APD) of all the algorithms considered are calculated and compared which is given in Table 2.

$S E_{k}=\sum_{p=1}^{P}\left|a_{p}-\hat{a}_{k p}\right|^{2}+\sum_{q=0}^{Q}\left|b_{q}-\hat{b}_{k q}\right|^{2}$

$$
A P D_{k}=\frac{1}{P+Q+1}\left[\sum_{p=1}^{P}\left|\frac{a_{k p}-\hat{a}_{k p}}{a_{k p}}\right|+\sum_{q=0}^{Q}\left|\frac{b_{k q}-\hat{b}_{k q}}{b_{k q}}\right| \times 100\right.
$$

Where $a_{p}$ and $b_{q}$ are the actual parameters and $\hat{a}_{k p}$ and $\hat{b}_{k q}$ denote the estimated parameters at the $k$ th sensor node. Estimated global parameter is defined as the mean of all the parameters estimated at every sensor node in the network. The SE and APD are illustrated as given in Equations 8 and 9.

From Table 2, it is observed that the non-cooperative LMS algorithm provides worst performance due to slow convergence as it is having very high values of SE and APD to that of the results from ILMS-IIR and DLMS-IIR algorithms. But in case of distributed diffusion LMS algorithms the performance is better as it provides less SE and APD than that of ILMS-IIR algorithm.

Table 2. Comparison of APD and SE by DLMS-IIR, ILMS-IIR, and non-cooperative LMS-IIR algorithms

\begin{tabular}{|l|l|l|l|l|l|l|}
\hline \multirow{2}{*}{ System Order } & \multicolumn{4}{l|}{ Mean APD across the Network } & \multicolumn{2}{l|}{ Mean SE across the Network } \\
\cline { 2 - 8 } & LMS-IIR & ILMS-IIR & DLMS-IIR & LMS-IIR & ILMS-IIR & DLMS-IIR \\
\hline 2nd & 6.5427 & 0.024 & 0.0081 & 0.0276 & $2.05 \mathrm{E}-08$ & $4.80 \mathrm{E}-09$ \\
\hline 3rd & 12.2401 & 0.0441 & 0.0104 & 0.0293 & $3.38 \mathrm{E}-08$ & $5.46 \mathrm{E}-09$ \\
\hline 4th & 29.4311 & 0.0719 & 0.0302 & 0.2252 & $8.45 \mathrm{E}-08$ & $1.67 \mathrm{E}-08$ \\
\hline
\end{tabular}

\subsection{Performance Analysis of Multihop Diffusion Algorithms (hDLMS) for Sparse Wireless Sensor Network}

Performance of diffusion LMS algorithm in less connected network is discussed here. Consider a sparse sensor network with 20 sensor nodes. The ILMS algorithm not possible here to estimate the systems parameter using distributed approach for a big network. Multihop diffusion LMS algorithm is used here for IIR systems parameter estimation (hDLMS-IIR).

The MSE and MSD simulation results are plotted in Figures 4, 5 and 6 for 2nd, 3rd and 4th order IIR system respectively. From the figure it is evident that the 2-hop hDLMSb algorithm perform well by providing less MSD and MSE performance with faster convergence compared to that of 1-hop DLMS algorithm in a sparse WSN. There is an improvement of $5 \mathrm{~dB}$ and $10 \mathrm{~dB}$ in MSE and MSD performance of 2-hop hDLMS algorithm over 1-hop algorithms respectively. 
Figure 4. Average MSE (a) and MSD (b) performances of 2nd order IIR system by DLMS-IIR, 2h-DLMS-IIR and NobCopLMS- IIR algorithms
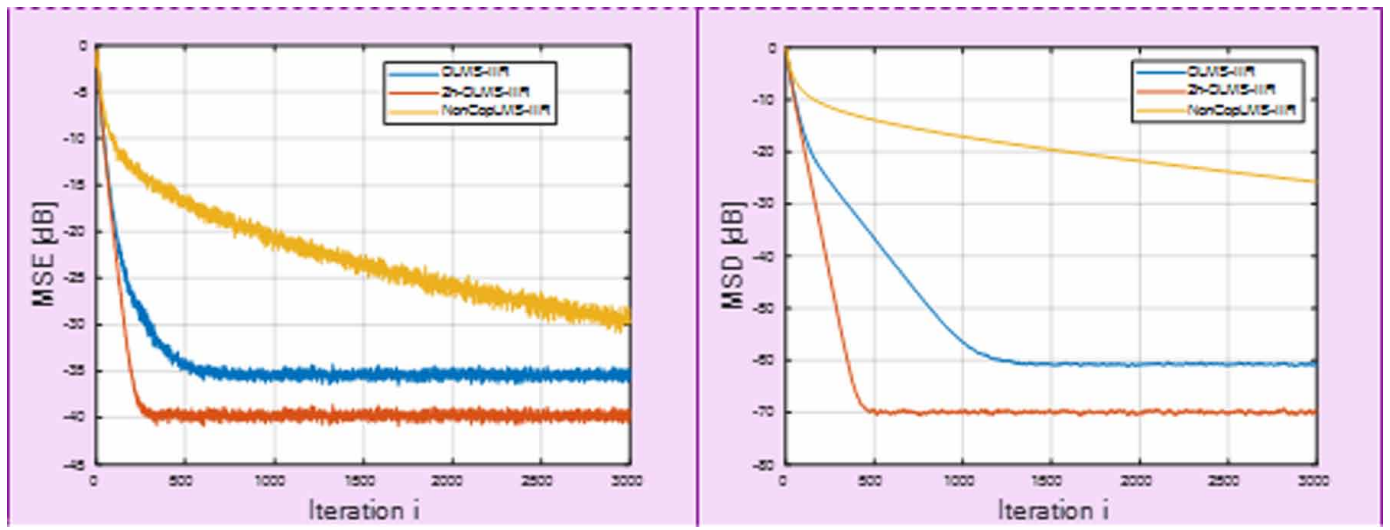

Figure 5. Average MSE (a) and MSD (b) performances of $3^{\text {rd }}$ order IIR system by DLMS-IIR, 2h-DLMS-IIR and NobCopLMS- IIR algorithms
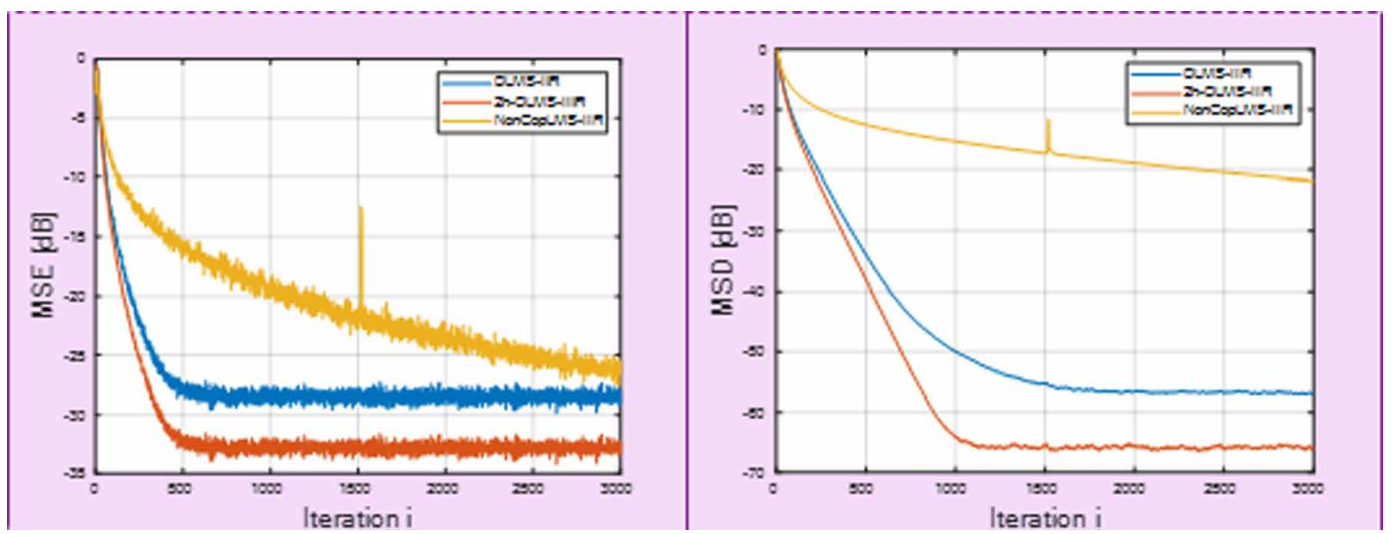

Figure 6. Average MSE (a) and MSD (b) performances of $4^{\text {th }}$ order IIR system by DLMS-IIR, $2 \mathrm{~h}$-DLMS-IIR and NobCopLMS- IIR algorithms
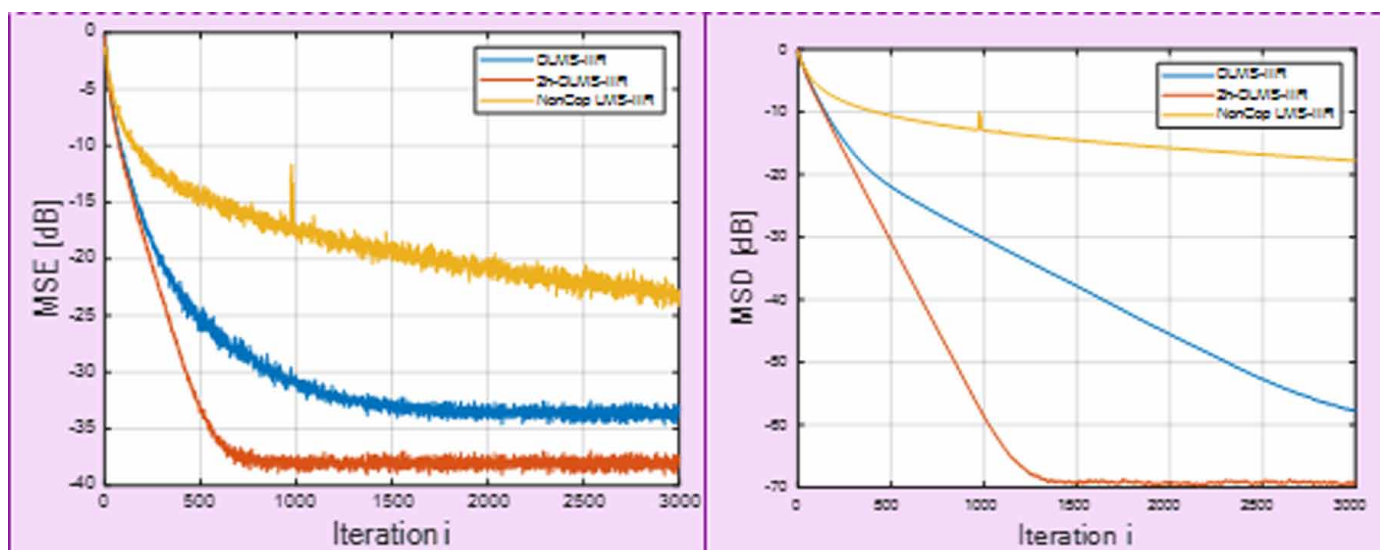


\section{CONCLUSION}

IIR system's parameter estimation by the distributed approach using diffusion LMS algorithm in sparse wireless sensor network has been studied here. Each sensor node is equipped with an adaptive IIR filter and nodes share information to other by using the diffusion mode of cooperation. The MSE and MSD are computed through simulations for $2^{\text {nd }}, 3^{\text {rd }}$ and $4^{\text {th }}$ order benchmark IIR systems and compared with that of Incremental LMS and non-cooperative LMS algorithms. The results inferred from simulations exhibit excellent performance of the DLMS algorithm as compared to that of noncooperative and incremental algorithms by providing less MSE and MSD. The multihop diffusion algorithm is proposed for sparse sensor network where the edge nodes fail to achieve good estimate by diffusion LMS algorithm. By doing this, the multihop diffusion LMS provides better MSE and MSD performance with minimum number of iterations. All the algorithms are simulated for a network with 20 sensor nodes. There is $10 \mathrm{~dB}$ and $5 \mathrm{~dB}$ improvement in steady state MSD and MSE performance respectively. In future a block diffusion multihop algorithm would be devised to reduce the communication overhead in the network by choosing proper block length. 


\section{REFERENCES}

Agrawal, N., Kumar, A., Bajaj, V., \& Singh, G. (2017). High order stable infinite impulse response filter design using cuckoo search algorithm. International Journal of Automation and Computing, 14(5), 589-602. doi:10.1007/s11633-017-1091-x

Arablouei, R., Werner, S., Huang, Y.-F., \& Doğançay, K. (2013). Distributed least mean-square estimation with partial diffusion. IEEE Transactions on Signal Processing, 62(2), 472-484. doi:10.1109/TSP.2013.2292035

Bazzi, W. M., Rastegarnia, A., \& Khalili, A. (2014). A quality-aware incremental lms algorithm for distributed adaptive estimation. International Journal of Automation and Computing, 11(6), 676-682. doi:10.1007/s11633014-0838-x

Cattivelli, F. S., \& Sayed, A. H. (2009). Diffusion LMS strategies for distributed estimation. IEEE Transactions on Signal Processing, 58(3), 1035-1048. doi:10.1109/TSP.2009.2033729

Dash, M., Panigrahi, T., \& Sharma, R. (2017). Distributed Parameter Estimation of IIR System using Diffusion Particle Swarm Optimization Algorithm. Journal of King Saud University - Engineering Sciences.

Dash, M., Panigrahi, T., \& Sharma, R. (2019). Robust Estimation of IIR System's Parameter using Modified Particle Swarm Optimization Algorithm. International Journal of Computational Intelligence Studies, 8(1/2).

Gharehshiran, O. N., Krishnamurthy, V., \& Yin, G. (2013). Distributed energy-aware diffusion least mean squares: Game-theoretic learning. IEEE Journal of Selected Topics in Signal Processing, 7(5), 821-836. doi:10.1109/ JSTSP.2013.2266318

Hu, W., \& Tay, W. P. (2015). Multi-hop diffusion LMS for energy-constrained distributed estimation. IEEE Transactions on Signal Processing, 63(15), 4022-4036. doi:10.1109/TSP.2015.2424206

Khalili, A., Rastegarnia, A., Bazzi, W. M., \& Rahmati, R. G. (2017). Incremental augmented complex adaptive IIR algorithm for training widely linear ARMA model. Signal, Image and Video Processing, 11(3), 493-500. doi:10.1007/s11760-016-0986-2

Kong, J.-T., Lee, J.-W., Kim, S.-E., Shin, S., \& Song, W.-J. (2017). Diffusion LMS algorithms with multi combination for distributed estimation: Formulation and performance analysis. Digital Signal Processing, 71, 117-130. doi:10.1016/j.dsp.2017.09.004

Li, Y., \& Thai, M. T. (2008). Wireless sensor networks and applications. Springer Science \& Business Media. doi:10.1007/978-0-387-49592-7

Majhi, B., \& Panda, G. (2013). Distributed and robust parameter estimation of IIR systems using incremental particle swarm optimization. Digital Signal Processing, 23(4), 1303-1313. doi:10.1016/j.dsp.2013.02.015

Mohapatra, U. M., \& Majhi, B. (2017). Distributed parameter estimation using incremental and diffusion differential evolution. In Handbook of Research on Wireless Sensor Network Trends, Technologies, and Applications (pp. 58-79). Hershey, PA: IGI Global.

Nayak, M., Panigrahi, T., \& Sharma, R. (2015). Distributed estimation using multi-hop adaptive diffusion in sparse wireless sensor networks. Paper presented at the 2015 International Conference on Microwave, Optical and Communication Engineering (ICMOCE). Academic Press. doi:10.1109/ICMOCE.2015.7489756

Nedic, A., \& Ozdaglar, A. (2009). Distributed subgradient methods for multi-agent optimization. IEEE Transactions on Automatic Control, 54(1), 48-61. doi:10.1109/TAC.2008.2009515

Panda, M., Gouda, B., \& Panigrahi, T. (2020). Distributed Online Fault Diagnosis in Wireless Sensor Networks. In Design Frameworks for Wireless Networks (pp. 197-221). Springer. doi:10.1007/978-981-13-9574-1_9

Panigrahi, T., Panda, M., \& Panda, G. (2016). Fault tolerant estimation in wireless sensor networks. Journal of Network and Computer Applications, 69, 27-39. doi:10.1016/j.jnca.2016.04.023

Panigrahi, T., Pradhan, P. M., Panda, G., \& Mulgrew, B. (2012). Block least mean squares algorithm over distributed wireless sensor network. Journal of Computer Networks and Communications.

Sayed, A. H. (2014a). Adaptation, learning, and optimization over networks. Foundations and Trends® in Machine Learning, 7(4-5), 311-801. 
Sayed, A. H. (2014b). Adaptive networks. Proceedings of the IEEE, 102(4), 460-497. doi:10.1109/ JPROC.2014.2306253

Xu, X., Wang, S., Mao, X., Tang, S., Xu, P., \& Li, X.-Y. (2009). Efficient data aggregation in multi-hop WSNS. Paper presented at the GLOBECOM 2009-2009 IEEE Global Telecommunications Conference. IEEE Press.

\begin{abstract}
Meera Dash has completed her M. Tech. in the year 2011 and is presently Asst. Prof. and pursuing her PhD in the Department of ECE, SOA Deemed to be University. She has 8 years experience in industry and about 10 years of teaching experience. Her area of research is distributed signal processing in WSN, signal Processing and Evolutionary algorithms. She has published several research publications in reputed International Journals and is a member IEEE and IEEE Communication Society.
\end{abstract}

Dr. Trilochan Panigrahi has completed his M.Tech. and PhD. degrees from NIT Rourkela. He has 15+ years of teaching undergraduate and post graduate Engineering students. His area of research is Distributedf signal processing in WSN, Array signal processing and Nano WSN. He has a number of research publications in reputed International Journals. He has successfully completed and is in the process of completing many SERB, DST, GOI funded research projects. He is a member of IEEE, IEEE signal processing Society, IEEE Communication Society, Institute of Engineers (India), Associate member, ISTE Life Member.

Renu Sharma $(P h D)$ is working as a Professor and Head of the Department Electrical Engineering under the Faculty of Engineering \& Technology, Siksha "O" Anusandhan University, Bhubaneswar. She received her Ph.D. in Electrical Engineering from Siksha "O" Anusandhan University, Bhubaneswar in 2014 and M. Tech in Electrical Engineering from Jadavpur University in 2006. At present, she has more than twenty years of teaching experience in the field of Electrical Engineering. She is Life Member IE (India), Member IET, Member IEEE, Life member ISTE, Life member ISSE. Her research areas are Smart Grid, Soft Computing, Solar Photovoltaic systems, Power system Scheduling, Evolutionary Algorithms and Wireless Sensor Networks. She has published more than 40 research papers in various reputed peer reviewed International Journals, Conferences and Book Chapters. She has served as reviewers from various reputed journal publishers such as Springer, IEEE, Elsevier and Inderscience. She is currently guiding eight Ph.D. scholar and one Postdoctoral fellow. She has organized several FDP and workshops sponsored by Typhoon HIL, AICTE, DST, etc.

Mihir Narayan Mohanty is currently working as a Professor in the Department of Electronics and Communication Engineering, Institute of Technical Education and Research (FET), Siksha 'O' Anusandhan (Deemed to be University), Bhubaneswar, Odisha. He has received his M. Tech degree in Communication System Engineering from the Sambalpur University, Sambalpur, Odisha and obtained his PhD degree in Applied Signal Processing. He is the fellow of IE (I), and IETE. Also, he is the member of many professional societies including IEEE, IET, etc. He has 25 years of teaching and research experience. He has published more than 300 papers in different journals, conferences including book chapters. He has authored two books. He is the successive reviewer of manuscripts from IEEE, Elsevier, Springer, IGI Global, etc. His areas of research interests include applied signal and image processing, wireless communication, antenna, and intelligent signal processing. 